\title{
Machine Learning: A Quantum Perspective
}

\author{
Aishwarya Jhanwar ${ }^{\text {a, } 1}$ and Manisha J. Nene ${ }^{\text {a }}$ \\ ${ }^{a}$ Department of Computer Science and Engineering, Defence Institute of Advanced \\ Technology, Pune-411025, Maharashtra
}

\begin{abstract}
Recently, increased availability of the data has led to advances in the field of machine learning. Despite of the growth in the domain of machine learning, the proximity to the physical limits of chip fabrication in classical computing is motivating researchers to explore the properties of quantum computing. Since quantum computers leverages the properties of quantum mechanics, it carries the ability to surpass classical computers in machine learning tasks. The study in this paper contributes in enabling researchers to understand how quantum computers can bring a paradigm shift in the field of machine learning. This paper addresses the concepts of quantum computing which influences machine learning in a quantum world. It also states the speedup observed in different machine learning algorithms when executed on quantum computers. The paper towards the end advocates the use of quantum application software and throw light on the existing challenges faced by quantum computers in the current scenario.
\end{abstract}

Keywords. Machine Learning, Quantum Computing, Quantum Machine Learning, Quantum Supremacy, Speedup

\section{Introduction}

In recent years, owing to increase number of datasets, machine learning has gained utmost success in transforming science and technology [1]. The aim of machine learning is to enable computers to act in a manner that barely involves any kind of human intervention and does not require the need of being programmed explicitly [2]. The vast ap-plications of machine learning ranging from predicting protein structure, drug discovery in biology [3] to black hole detection [4], wave analysis in physics [5] to speech recognition, self-driving cars, etc. in computers exhibits a remarkable contribution in today's scenario. However, with this ever-growing size of dataset, it has posed certain challenges in this field. With the Moore's law moving towards its extinction, we might probably attain a peak where current computational methods would not suffice handling of such humongous datasets [6].

This motivated to use the concept of quantum computing which is based on quantum mechanics. The application of quantum mechanics in the discipline of information processing is termed as Quantum Information Processing [7]. By exploiting the properties of quantum computing such as entanglement and superposition, quantum computers could efficiently solve some problems that are perceived as hard problems for classical computers [8,9,10].The applications developed in the field of machine learning using quantum computing algorithms exceeds the capabilities of any classical computer achieving quantum supremacy. A successful demonstration of quantum supremacy would prove that engineered quantum systems can outperform the most advanced classical computers [11].

\footnotetext{
${ }^{1}$ Aishwarya Jhanwar,Defence Institute of Advanced Technology, Pune, Inda Email: aishwaryajhanwar2001@gmail.com.
} 
This paper aims to provide technical insights of integrating quantum computing with machine learning that will prove beneficial to readers of both the communities i.e., of machine learning as well as of quantum computing.

\section{Classical Machine Learning}

Machine learning, is a sub-discipline of artificial intelligence where learning from data is involved. In 1959, Arthur Samuel described machine learning as "a field of study that gives computers the ability to learn without being explicitly programmed" [12]. Machine learning is used to find hidden structures and interesting patterns from given dataset. The intrinsic value of data can be augmented with machine learning. This value can be fetched in different manner depending upon the nature of the dataset available. Broadly, the term learning in machine learning can be divided into three types: Supervised, Unsupervised and Reinforcement Learning. In Supervised Learning a computer is fed with input-output data pairs that helps to infer an algorithm and predict the output. It is generally used for classification of data based on the labels. Examples of supervised learning classifier includes K Nearest Neighbors, Decision Tree, Naive Bayes and Random Forest models. In Unsupervised Learning input-output data pairs are not given to the machine, rather the system is fed with unlabeled data and hence it builds an algorithm to find the patterns. It is used for clustering of data. Some of the examples are Apriori, K-means and Principal Component Analysis. Reinforcement Learning uses software agents to earn cumulative rewards and thus proceed to train the algorithm in a certain manner. In all these learning methods, the indispensable role is of training phase. Training phase is often considered as most costly phase of any machine learning process. When dealing with extremely large datasets efficient training methods plays a vital role.

Irrespective of what learning method is adopted, an optimal machine learning algorithm is the one which produces a minimum error rate by consuming minimum resources. Due to ever increasing rate of data, current machine learning systems are nudging the limit of classical computational resources. Challenges lies in the problem of finding an optimal solution to any problem, that helps to minimize the complexity class of the problem [13]. This is where there arises a need to shift to quantum computing.

\section{Quantum Computing}

Quantum computing deals with the problem of processing, storing and transferring information which is encoded in quantum mechanical systems. This mode of information is termed as quantum information. Quantum information is processed by acting upon quantum systems. Basic quantum computing concepts that influence any algorithm to perform in an efficient manner [14].

\subsection{Qubit}

Quantum bits or qubit is the fundamental unit of processing information in quantum systems analogous to a bit in classical systems. A qubit can exist in one or more than one state simultaneously. However, the information content stored in a qubit that is 
equivalent to a single bit in classical medium. It is defined as linear superposition of states.

$$
|\psi\rangle=\alpha|\mathbf{0}\rangle+\beta|\mathbf{1}\rangle \quad \alpha, \beta \in \mathrm{C}
$$

where, $|\alpha|^{2}+|\beta|^{2}=1$. The complex numbers $\alpha$ and $\beta$ are probabilities of the basic states of $|0\rangle$ and $|1\rangle$, and $|0\rangle$ and $|1\rangle$ represent the orthogonal states.

\subsection{Superposition}

A qubit can be in a state of $|0\rangle$ and $|1\rangle$ at the same time. This implies that to store two bits worth of value only one qubit is required. On similar lines for 4 bits, 2 qubits will be required and hence, $2^{N}$ Classical Bits can be stored on $N$ Qubits. This means that a particle has an ability to exist in multiple quantum states and when the measurement is performed, it undergoes certain changes resulting in a probabilistic value, thereby losing its individuality $[15,16]$.

\subsection{Entanglement}

In a multi qubit system, qubits exist in a manner that they lose their individuality. It means the property of one qubit is connected to the property of another qubit. Thus, this property helps the qubits to be correlated with each other despite of being separated by large physical distances $[17,18]$.

\subsection{No Cloning Theorem}

This theorem states that it is not possible to create an identical copy of an arbitrary unknown quantum state. It helps to infer that once a measurement is performed, it is not sure to get the same information after another measurement being performed on an already measured state.

Quantum measurement depends on the wave function collapse. It occurs when a wave function, initially in a superposition of several eigen states reduces to a single eigen state due to interaction with the external world. This leads to measurement of a Quantum state which is probabilistic in nature.

These paramount features offered by quantum computers are required for highspeed computing which paves way to delve deeper and integrate machine learning with quan-tum computing.

\section{Quantum Machine Learning}

Quantum Machine Learning (QML) is the intersection of machine learning process with the concepts of quantum computing. In QML, quantum algorithms are developed to solve intricate problems of machine learning utilizing the potency of quantum computing. This is achieved by enabling expensive subroutines of classical algorithms to be executed on a quantum computer. The properties of quantum computing such as superposition induces parallelism in quantum computers which allows to evaluate function on many inputs in machine learning algorithms simultaneously. Entanglement provides a mechanism for improving the storage capacity as well as retrieving 
corrupted or incomplete information $[19,20]$. These properties thus provide significant speedup of any computation evaluated on the basis of complexity [21]. By speedup it means the advantages obtained in run time by any quantum algorithm as compared to the classical methods used for the same task [22]. Table 1 gives a general overview of the different machine learning algorithms and their speedups when executed on quantum computers. The subroutines used by these algorithms uses the properties of quantum computing as discussed in section 3 to enhance their efficiency. It will thus help to understand how quantum computers can revolutionize machine learning.

Table 1. Speed up of machine learning algorithms in quantum environment

\begin{tabular}{|c|c|}
\hline Algorithm & Speedup \\
\hline Associative Memory [23] & Exponential improvement in capacity \\
\hline Boosting [24] & Quadratic \\
\hline Divisive Clustering [25] & Exponential \\
\hline Gradient Descent [26] & Exponential \\
\hline K-Means [21,27] & Quadratic \\
\hline K-Medians [25] & Quadratic \\
\hline K Nearest Neighbors [28] & Exponential improvement in capacity \\
\hline Pattern Recognition [29] & Exponential \\
\hline Principal Components Analysis [30] & Exponential \\
\hline Q-means [31] & Exponential \\
\hline Rupport Vector Machines [33] & Exponential \\
\hline
\end{tabular}

\section{Quantum Application Software and Challenges}

The gap between the computational models and its actual implementation has been narrowed down to a great extent with the development of open-source quantum software platforms around the globe. Some leading-edge quantum platforms like QISKIT [34], DWave [35], ProjectQ [36], Forest [37], Strawberry Fields [38], Quantum Development Kit [39] and Cirq [40]. allow these algorithms to be implemented on real quantum computers which can be accessed through cloud-based services or quantum simulators which runs on classical machines [41]. Thus, these software makes it possible to implement machine learning in quantum environment and experience the enhanced outcomes.

QML poses a great challenge in the field of Quantum Information Processing as quantum computing is still in its infancy stage of development. Qubits are affected by various disturbances such as vibrations, electromagnetic waves, temperature 
fluctuations, cosmic rays, etc. that are induced by the effects of quantum mechanics which are extremely sensitive to external conditions. Thus, the fragile nature of qubits makes them pone to high errors. The researchers are working in the field of mitigating errors induced due to noise and fragile nature of quantum computers [42]. Another challenge is the limited availability of quantum computers. As of now rigorous study is required to decide upon how many logical qubits are required by quantum computers to exceed the capabilities of classical computers, that are extremely powerful [43].

\section{Conclusion}

The study in this paper reveals that with the existence of quantum supremacy a drastic change will be seen in the field of machine learning. The immense challenges require different approaches of quantum computing to be devised and investigated to enhance machine learning methods. The properties leveraged by quantum computers helps to achieve considerable speedups in the field of machine learning. Although QML has gained a lot of awareness amongst quantum scientists and machine learning researchers, yet in order to gain maximum benefits areas which remain unfolded may be explored and delved deeper.

\section{References}

[1] Silver D et al. 2016 Mastering the game of Go with deep neural networks and tree search. Nature 529, 484-489. (doi:10.1038/nature16961)

[2] Ian Goodfellow, Yoshua Bengio, and Aaron Courville. Deep learning. MIT press, 2016.

[3] Mohammed AlQuraishi. Alphafold at casp13. Bioinformatics, 35(22):4862-4865, 2019.

[4] Benjamin P Abbott, Richard Abbott, TD Abbott, MR Abernathy, Fausto Acernese, Kendall Ackley, Carl Adams, Thomas Adams, Paolo Addesso, RX Adhikari, et al. Observation of gravitational waves from a binary black hole merger.Physical review letters, 116(6):061102, 2016.

[5] Louis-Franc, ois Arsenault, O Anatole von Lilienfeld, and Andrew J Millis. Machine learning for many-body physics: efficient solution of dynamical mean-field theory. arXiv preprint arXiv:1506.08858, 2015.

[6] Markov IL. 2014 Limits on fundamental limits to computation. Nature 512, 147-154.

[7] SM. Barnett, "Introduction to Quantum Information", School of Physics and Astronomy, University of Glasgow, Glasgow G12 8QQ, UK, Oxford University Press, pp. 11-33

[8] Shor PW. 1997 Polynomial-time algorithms for prime factorization and discrete logarithms on a quantum computer. SIAM J. Comput. 26, 1484-1509. (doi:10.1137/S0097539795293172)

[9] Nene M.J., Upadhyay G. (2016) Shor's Algorithm for Quantum Factoring. In: Choudhary R., Mandal J., Auluck N., Nagarajaram H. (eds) Advanced Computing and Communication Technologies. Advances in Intelligent Systems and Computing, vol 452. Springer, Singapore. https://doi.org/10.1007/ 978-981-10-1023-1 33

[10] Van Dam W, Hallgren S, Ip L. 2006 Quantum algorithms for some hidden shift problems. SIAM J. Comput. 36, 763-778. (doi:10.1137/S009753970343141X)

[11] Neill C, Roushan P, Kechedzhi K, Boixo S, Isakov SV, Smelyanskiy V, Megrant A, Chiaro B, Dunsworth A, Arya K, Barends R, Burkett B, Chen Y, Chen Z, Fowler A, Foxen B, Giustina M, Graff R, Jeffrey E, Huang T, Kelly J, Klimov P, Lucero E, Mutus J, Neeley M, Quintana C, Sank D, Vainsencher A, Wenner J, White TC, Neven H, Martinis JM. A blueprint for demonstrating quantum supremacy with su-perconducting qubits. Science. 2018 Apr 13;360(6385):195-199. doi: 10.1126/science.aao4309. PMID: 29650670.

[12] Arthur L Samuel. Some studies in machine learning using the game of checkers. IBM Jour- nal of research and development, 44(1.2):206-226, 2000.

[13] Schuld, Maria \& Sinayskiy, Ilya \& Petruccione, Francesco. (2014). An introduction to quantum machine learning. Contemporary Physics. 56. 10.1080/00107514.2014.964942.

[14] Sharma, MK, Nene, MJ. "Dual factor third-party biometric-based authentication scheme using quantum one time passwords". Security and Privacy. 2020; 3:e129.https://doi.org/10. $1002 /$ spy 2.129 
[15] G. Upadhyay and M. J. Nene, "One time pad generation using quantum superposition states," 2016 IEEE International Conference on Recent Trends in Electronics, Information \& Communication Technology (RTEICT), Bangalore, 2016, pp. 1882-1886, doi: 10.1109/RTEICT.2016.7808162.

[16] M. Gupta and M. J. Nene, "Random Sequence Generation using Superconducting Qubits," 2021 Third International Conference on Intelligent Communication Technologies and Virtual Mobile Networks (ICICV), Tirunelveli, India, 2021, pp. 640-645, doi: 10.1109/ICICV50876.2021.9388495.

[17] M. Gupta and M. J. Nene, "Quantum Computing: An Entanglement Measurement," 2020 IEEE International Conference on Advent Trends in Multidisciplinary Research and Innovation (ICATMRI), 2020, pp. 1-6, doi: 10.1109/ICATMRI51801.2020.9398441.

[18] P. Nema and M. J. Nene, "Pauli Matrix based Quantum Communication Protocol," 2020 IEEE International Conference on Advent Trends in Multidisciplinary Research and Innovation (ICATMRI), 2020, pp. 1-6, doi: 10.1109/ICATMRI51801.2020.9398393.

[19] Adcock J et al. 2015 Advances in quantum machine learning. (http://arxiv.org/abs/ 1512.02900).

[20] Biamonte J, Wittek P, Pancotti N, Rebentrost P, Wiebe N, Lloyd S. 2017 Quantum machine learning. Nature 549, 195-202. (doi:10.1038/nature23474)

[21] Lloyd, S., Mohseni, M. \& Rebentrost, P. Quantum algorithms for supervised and unsupervised machine learning. arXiv:1307.0411 (2013).

[22] Ciliberto, Carlo \& Herbster, Mark \& Ialongo, Alessandro \& Pontil, Massimiliano \& Rocchetto, An-drea \& Severini, Simone \& Wossnig, Leonard. (2017). Quantum machine learning: A classical per-spective. Proceedings of the Royal Society A: Mathematical, Physical and Engineering Science. 474. 10.1098/rspa.2017.0551.

[23] D. Ventura and T. Martinez, "Quantum Associative Memory," Int. J. Mod. Phys. B, vol. 17, no. 12, pp. 2447-2472, Jul. 1998.

[24] H. Neven, V. S. Denchev, G. Rose, and W. G. Macready, "Training a Large Scale Classifier with the Quantum Adiabatic Algorithm,” pp. 1-11, 2009.

[25] E. A"imeur, G. Brassard, and S. Gambs, "Quantum clustering algorithms," in Proceedings of the 24th international conference on Machine learning - ICML '07, 2007, vol. 227, pp. 1-8.

[26] I. Kerenidis and A. Prakash, "Quantum gradient descent for linear systems and least squares," 2017.

[27] Khan, Sumsam Awan, Ahsan Javed Vall Llosera Gemma 2019 K Means Clustering on Noisy Intermediate Scale Quantum Computers.

[28] N. Wiebe, A. Kapoor, and K. M. Svore, "Quantum algorithms for nearest neighbor methods for supervised and unsupervised learning," Quantum Inf. Comput., vol. 15, no. 3-4, pp. 318-358, 2015.

[29] C. A. Trugenberger, "Quantum Pattern Recognition," in Invited Talk at the 1st Feynman Festival, Univ. of Maryland, College Park, August 2002, 2002.

[30] S. Lloyd, M. Mohseni, and P. Rebentrost, "Quantum principal component analysis," Nat. Phys., vol. 10, no. 9, pp. 631-633, 2014.

[31] I. Kerenidis, J. Landman, A. Luongo, and A. Prakash, "q-means: A quantum algorithm for unsupervised machine learning," 2018.

[32] I. Kerenidis and A. Prakash, "Quantum recommendation system," Leibniz Int. Proc. Informatics, LIPIcs, vol. 67, pp. 1-22, 2017.

[33] D. Anguita, S. Ridella, F. Rivieccio, and R. Zunino, "Quantum optimization for training support vector machines," Neural Networks, vol. 16, no. 5-6, pp. 763-770, 2003.

[34] Qiskit https://qiskit.org/ last accessed 14 April 2021

[35] DWave The Quantum Computing Company https://www.dwavesys.com/quantum-computing last accessed 14 April 2021

[36] ProjectQ https://projectq.ch/ last accessed 14 April 2021

[37] Rigetti https://www.rigetti.com/ last accessed 14 April 2021

[38] Strawberry Fields https://strawberryfields.ai/ last accessed 14 April 2021

[39] Q\# and The Quantum Development Kit https://azure.microsoft.com/enus/resources/developmentkit/quantum-computing/ last accessed 14 April 2021

[40] Cirq https://quantumai.google/cirq last accessed 14 April 2021

[41] LaRose, R. (2019). Overview and Comparison of Gate Level Quantum Software Platforms. Quantum, 3, 130.

[42] Fowler, A. G.; Mariantoni, M.; Martinis, J. M. \& Cleland, A. N. "Surface Codes: Towards Practical Large-scale Quantum Computation”. Physical Review A, 86(3), 32324 (2012)

[43] Muller, R. P. \& Blume-Kohout, R. "The Promise of Quantum Simulation”. ACS Nano (2015) 